\title{
Ambient RF Energy Harvesting with Non-Linearities in Large-Scale Networks
}

\author{
Constantinos Psomas and Ioannis Krikidis \\ IRIDA Research Centre for Communication Technologies \\ Department of Electrical and Computer Engineering, University of Cyprus, Cyprus \\ Email: \{psomas, krikidis\}@ucy.ac.cy
}

\begin{abstract}
Ambient radio-frequency (RF) energy harvesting is a potential solution for charging low-powered devices, as a result of its cost effectiveness and pervasiveness. In this paper, we deal with devices which are underlaid in a wireless communication network and harvest energy from the ambient RF transmitted signals. The devices are equipped with an array of multiple rectifying antennas, combined either at the $\mathrm{RF}$ or the DC domain, to increase the harvesting efficiency. A large-scale analysis is undertaken, with the use of stochastic geometry. We take into account the non-linearities of the RF energy harvesting process and derive closed-form analytical expressions for the average harvested energy for both array architectures. Our results show the importance of considering non-linearities, mainly in terms of the network's density and the size of the rectifying antenna array but also for other network parameters.
\end{abstract}

Index Terms-Wireless power transfer, non-linear energy harvesting, Poisson point process.

\section{INTRODUCTION}

The concept of harvesting energy from electromagnetic radiation is gradually becoming more and more compelling [1]. This is due to recent advances, both practical and theoretical, in the field of wireless power transfer (WPT), which have shown its feasibility as well as the benefits emerging from this technology. Energy harvesting from radio-frequency (RF) signals, transmitted by a dedicated or an ambient RF source, is achieved with the employment of a rectifying antenna (rectenna) at the receiver. A dedicated RF source can transfer energy more efficiently, compared to an ambient source, through the use of signal processing techniques such as energy beamforming. However, the future deployment of ultra-dense heterogeneous wireless networks, will lead to a substantial increase in ambient RF signals, which can be exploited for WPT.

Ambient RF energy harvesting has been studied extensively in the literature for various scenarios [2]-[8]. In [2], the authors consider a battery-less sensor which harvests energy from ambient RF sources and uses that energy to power the uplink transmission; it is shown that the sensors perform better when the spatial distribution of the ambient sources has a stronger repulsion. The work in [3] considers a multi-tier

This work was co-funded by the European Regional Development Fund and the Republic of Cyprus through the Research and Innovation Foundation, under the project POST-DOC/0916/0256 (IMPULSE), and has received funding from the European Research Council (ERC) under the European Union's Horizon 2020 research and innovation programme (Grant agreement No. 819819). cellular network, where the users power their uplink transmission, by harvesting energy from the base stations' transmitted signals; the authors show how the system's parameters affect its performance. A cellular network is also studied in [4], where the devices harvest ambient RF energy and use it to operate both the downlink and the uplink; the authors derive the joint uplink/downlink probability.

The works [5] and [6] deal with device-to-device (D2D) communications. In [5], the D2D transmitters harvest energy from a dedicated power beacon and from the ambient RF sources of the network in order to establish D2D communications. In [6], a cellular network is underlaid with a D2D network, where the D2D communications are powered by the energy obtained from the cellular downlink transmissions. Ambient energy harvesting has also been studied in the context of backscatter communications [7], [8]. The authors in [7] consider a cognitive network, where the secondary transmitter backscatters ambient signals to the secondary transmitter; optimization problems are formulated and the overall transmission rate of the secondary system is maximized. Finally, a hybrid D2D scenario combining both ambient backscatter and wireless powered communications is considered in [8], and the authors show the benefits that can be achieved by such a scenario.

Despite the many works that exist on ambient RF energy harvesting from a large-scale point-of-view, to the best of our knowledge, none of these take into account the nonlinearities of the RF energy harvesting process based on the physics of the diode [1]. Moreover, the effect of fading due to the non-linearities [9], has not been studied for multi-user environments. These limitations motivate the work presented in this paper. Specifically, we consider a wireless network consisting of multiple transmitters and receivers, as well as a set of devices which harvest energy from the ambient RF transmitted signals. Each device is equipped with a rectenna array consisting of multiple elements, in order to enhance the harvesting efficiency. A general Nakagami fading model but also the non-linearities of energy harvesting are taken into consideration. We derive closed-form analytical expressions for the average harvested energy of two fundamental rectenna array architectures. Our results verify the benefits that emerge from considering the non-linearities in terms of the network's parameters and show the significant gains that can be achieved by the employment of rectenna arrays. 


\section{SYSTEM MODEL}

\section{A. Topology}

We consider a wireless network with multiple randomly deployed transmitters/receiver pairs (e.g. a cellular or ad-hoc network), underlaid with a set of low-powered devices (e.g. sensors) which harvest RF ambient energy in order to power their operations. The transmitters are assumed to be spatially distributed according to a homogeneous Poisson point process (PPP) $\Phi=\left\{x_{k}\right\}, k \geq 1$, of density $\lambda$, where $x_{k}$ denotes the coordinates of the $k$-th transmitter. All transmitters are equipped with a single antenna and transmit with fixed power $P_{t}$. Each low-powered device employs a rectenna array of $L$ antenna elements [10]. All antennas are considered to be omnidirectional. We are interested in the energy harvesting performance of a device and so we focus on the typical device. Specifically, we consider the typical device located at the origin of a circular area, denoted by $\mathcal{D}$, and modeled as a disc of radius $\rho$.

\section{B. Channel Model}

All wireless links are assumed to suffer from both smallscale block fading and large-scale path-loss effects. We consider Nakagami fading with integer parameter $m, m \geq 1$, and so the power of the channel fading is a normalized Gamma random variable with shape parameter $m$ and scale parameter $1 / m$. We denote by $h_{k, i}$ the channel coefficient for the link between the $k$-th transmitter and the $i$-th receive antenna of the typical device. The path-loss model assumes that the received power is proportional to $1 /\left(1+d_{k}^{\alpha}\right)$ where $d_{k}$ is the Euclidean distance from the origin to the $k$-th transmitter and $\alpha>2$ is the path-loss exponent. The transmitted RF signal from the $k$-th transmitter is

$$
s_{k}(t)=\sqrt{2 P_{t}} \Re\{x(t) \exp (\jmath 2 \pi f t)\},
$$

where $P_{t}=\mathbb{E}\left\{s^{2}(t)\right\}$ is the transmit power, $f$ denotes the carrier frequency, and $x(t)$ is a modulated energy signal with $x(t) \sim \mathcal{N}(0,1)^{1}$. Therefore, the received signal from the $k$-th transmitter at the typical device's $i$-th antenna is given by

$$
\begin{aligned}
y_{k, i}(t) & =\sqrt{\frac{2 P_{t}}{1+d_{k}^{\alpha}}} \Re\left\{h_{k, i}(t) x(t) \exp (\jmath 2 \pi f t)\right. \\
& =\sqrt{\frac{2 P_{t}}{1+d_{k}^{\alpha}}}\left|h_{k, i}(t)\right| x(t) \cos \left(2 \pi f t+\theta_{k, i}(t)\right),
\end{aligned}
$$

where $\left|h_{k, i}(t)\right|$ is a Nakagami distributed random variable with parameter $m$ and $\theta_{k, i}(t)$ is the phase at the $i$-th antenna.

\section{Energy Harvesting}

A device attempts to harvest energy from the ambient RF signals in the network. The received aggregate signal at each antenna is then converted to a direct-current (DC) output with the use of a rectifier. A rectifier is a basic circuit, usually consisting of a diode (e.g. a Schottky diode) and a passive low

\footnotetext{
${ }^{1}$ Other distributions for $x(t)$ could also be considered such as complex circularly symmetric Gaussian.
}

pass filter (LPF) [1]. The output current of the diode from a received signal $y(t)$ is given by

$$
I(t)=I_{s}\left(\exp \left(\frac{y(t)}{\mu V_{T}}\right)-1\right)=I_{s} \sum_{j=1}^{\infty} \frac{1}{j !}\left(\frac{y(t)}{\mu V_{T}}\right)^{j},
$$

where $I_{s}$ denotes the reverse saturation current of the diode, $\mu$ is an ideality factor which is a function of the operating conditions and physical contractions, and $V_{T}$ is the thermal voltage; the resulting expression in (3) follows from the Taylor series expansion of an exponential function [1]. By taking the expectation of (3), preserves the DC component of the diode's output current. Therefore, by only keeping the second and fourth order term from the expectation of (3), the total harvested energy $\mathrm{E}(t)$ is a non-linear function of $I(t)$, written as [9]

$$
\mathrm{E}(t)=\gamma_{2} \mathbb{E}\left[y(t)^{2}\right]+\gamma_{4} \mathbb{E}\left[y(t)^{4}\right]
$$

where

$$
\gamma_{i}=\frac{I_{s}}{i !\left(\mu V_{T}\right)^{i}} .
$$

We assume that each device is equipped with a rectenna array consisting of $L$ antenna elements in order to boost the rectification process and increase its efficiency. The interconnection of the $L$ antenna elements can be performed either in the DC domain or the RF domain [10]. The RF combiner amalgamates all $L$ signals from each antenna element before the rectification process. By assuming the rectenna array aligns the phases of the incoming signals to $\theta_{k}(t)$, the combined received signal from the $k$-th transmitter is given by

$$
\begin{aligned}
y_{k}^{\mathrm{RF}}(t) & =\sqrt{\frac{2 P_{t}}{1+d_{k}^{\alpha}}} \sum_{i=1}^{L} \Re\left\{h_{k, i}(t) x(t) \exp (\jmath 2 \pi f t)\right\} \\
& =\sqrt{\frac{2 P_{t}}{1+d_{k}^{\alpha}}} c_{k}(t) x(t) \cos \left(2 \pi f t+\theta_{k}(t)\right),
\end{aligned}
$$

where $c_{k}(t)=\sum_{i=1}^{L}\left|h_{k, i}(t)\right|$ is the sum of $L$ Nakagami random variables. Therefore, assuming there are $K$ transmitters in the network, the harvested energy $\mathrm{E}_{\mathrm{RF}}(t)$ with the RF combiner is

$$
\mathrm{E}_{\mathrm{RF}}(t)=\gamma_{2} \mathbb{E}\left[\left(\sum_{k=1}^{K} y_{k}^{\mathrm{RF}}(t)\right)^{2}\right]+\gamma_{4} \mathbb{E}\left[\left(\sum_{k=1}^{K} y_{k}^{\mathrm{RF}}(t)\right)^{4}\right],
$$

where $y_{k}^{\mathrm{RF}}(t)$ is given by (6). On the other hand, for the DC combiner, the energy harvesting operation from the elements is mutually independent since each has its own rectification circuit. Thus, in this case, the harvested energy $\mathrm{E}_{\mathrm{DC}}(t)$ is

$$
\begin{aligned}
& \mathrm{E}_{\mathrm{DC}}(t)=\sum_{i=1}^{L}\left(\gamma_{2} \mathbb{E}\left[\left(\sum_{k=1}^{K} y_{k, i}(t)\right)^{2}\right]\right. \\
&\left.+\gamma_{4} \mathbb{E}\left[\left(\sum_{k=1}^{K} y_{k, i}(t)\right)^{4}\right]\right),
\end{aligned}
$$

where $y_{k, i}(t)$ is given by (2). 


\section{Average Harvested Energy}

In this section, we derive the analytical expressions for the average harvested energy at a wireless powered device. We first state the following, which will assist in the derivation of the final expressions. Suppose there are $K$ transmitters in the disc $\mathcal{D}$. Since $K$ is Poisson distributed, its probability mass function (PMF) is given by

$$
p(K)=\frac{1}{K !} \exp (-\lambda|\mathcal{D}|)(\lambda|\mathcal{D}|)^{K},
$$

where $|\mathcal{D}|=\pi \rho^{2}$. Moreover, the $n$-th moment of the path-loss from the origin of $\mathcal{D}$ to the $k$-th transmitter is given by

$$
\begin{aligned}
\bar{r}_{n}=\mathbb{E}\left[\frac{1}{\left(1+d_{k}^{\alpha}\right)^{n}}\right] & =\int_{0}^{\rho} \frac{1}{\left(1+r^{\alpha}\right)^{n}} f_{r}(x) d r d \phi \\
& ={ }_{2} F_{1}\left(n, \frac{2}{\alpha} ; 1+\frac{2}{\alpha} ;-\rho^{\alpha}\right),
\end{aligned}
$$

where $f_{r}(x)=2 r / \rho^{2}$ is the probability density function of the distance to the origin, ${ }_{2} F_{1}(\cdot, \cdot ; \cdot ; \cdot)$ is the Gauss hypergeometric function [11] and (10) follows from the transformation $r^{\alpha} \rightarrow x$ and using [11, 3.194.1].

The main expressions for the average harvested energy obtained with the RF and DC combiners, are given in the following two propositions.

Proposition 1. The average harvested energy $E_{R F}$ achieved by the RF combiner is

$$
\begin{array}{r}
E_{R F}=P_{t} \sum_{K=0}^{\infty} p(K) K\left(\gamma_{2} \epsilon_{2} \bar{r}_{1}+3 P_{t} \gamma_{4} \epsilon_{2}^{2}(K-1) \bar{r}_{1}^{2}\right. \\
\left.+\frac{9}{2} P_{t} \gamma_{4} \epsilon_{4} \bar{r}_{2}\right),
\end{array}
$$

where

$$
\epsilon_{2}=L\left(\frac{w_{1}^{2}}{m}(L-1)+1\right),
$$

and

$$
\begin{aligned}
\epsilon_{4}=\frac{L}{m^{2}}\left(w_{4}\right. & +(L-1)\left(3 w_{2}^{2}+4 w_{3} w_{1}\right) \\
& \left.+(L-1)(L-2)\left(6 w_{2} w_{1}^{2}+(L-3) w_{1}^{4}\right)\right),
\end{aligned}
$$

with

$$
w_{i}=\frac{\Gamma(m+i / 2)}{\Gamma(m)} .
$$

Proof. See Appendix A.

Proposition 2. The average harvested energy $E_{D C}$ achieved by the DC combiner is

$$
\begin{aligned}
E_{D C}=P_{t} L \sum_{K=0}^{\infty} p(K) K\left(\gamma_{2} \bar{r}_{1}+\right. & 3 P_{t} \gamma_{4}(K-1) \bar{r}_{1}^{2} \\
& \left.+\frac{9}{2} P_{t} \gamma_{4} \frac{m+1}{m} \bar{r}_{2}\right) .
\end{aligned}
$$

Proof. See Appendix B.
It is worth noting that the first term in both (11) and (15) characterizes the performance of the linear model, i.e. the second order term in (4). In the following corollary, we consider the case of Rayleigh fading, i.e. $m=1$, and one antenna element, i.e. $L=1$; note that in this case $\mathrm{E}_{\mathrm{RF}}=\mathrm{E}_{\mathrm{DC}}$, since no combiner is employed.

Corollary 1. The average harvest energy $E$ achieved over Rayleigh fading and with $L=1$ is

$$
E=P_{t} \sum_{K=0}^{\infty} p(K) K\left(\gamma_{2} \bar{r}_{1}+3 P_{t} \gamma_{4}(K-1) \bar{r}_{1}^{2}+9 P_{t} \gamma_{4} \bar{r}_{2}\right) .
$$

In what follows, we evaluate the energy harvesting performance for special case scenarios. We consider three cases, namely, ultra-dense cells (large $\lambda$ ), large cells $(\rho \rightarrow \infty)$, and deterministic fading channels (large $m$ ). These cases are provided in the following remarks.

Remark 1. For ultra-dense cells, the average harvested energy achieved by the RF combiner is approximated by

$$
\lim _{\lambda \rightarrow \infty} E_{R F} \approx 3 P_{t}^{2} \sum_{K=0}^{\infty} p(K) K^{2} \gamma_{4} \epsilon_{2}^{2} \bar{r}_{1}^{2},
$$

and the average harvested energy achieved by the DC combiner is approximated by

$$
\lim _{\lambda \rightarrow \infty} E_{D C} \approx 3 P_{t}^{2} L \sum_{K=0}^{\infty} p(K) K^{2} \gamma_{4} \bar{r}_{1}^{2} .
$$

Proof. The expressions are derived directly from Propositions 1 and 2. For very large $\lambda$, the number of transmitters $K$ also becomes very large. In this case, the first and third terms in both (11) and (15), become very small and thus we are left with the second term which dominates the average harvested energy.

From Remark 1, we can deduce that the RF combiner always outperforms the DC combiner, since $\epsilon_{2}^{2}>L$. Furthermore, $\epsilon_{2}^{2}=L$ only when $L=1$, i.e. when no combiner is employed.

Remark 2. For large cell radius $\rho$, the average harvested energy achieved by the RF combiner is

$$
\begin{aligned}
& \lim _{\rho \rightarrow \infty} E_{R F}=\frac{\lambda \pi^{2}}{\alpha^{2}} P_{t} \csc \left(\frac{2 \pi}{\alpha}\right) \\
& \times\left(2 \alpha \gamma_{2} \epsilon_{2}+12 \gamma_{4} \epsilon_{2}^{2} \lambda \pi^{2} P_{t} \csc \left(\frac{2 \pi}{\alpha}\right)+9(\alpha-2) P_{t} \gamma_{4} \epsilon_{4}\right),
\end{aligned}
$$

and the average harvested energy achieved by the DC combiner is

$$
\begin{aligned}
& \lim _{\rho \rightarrow \infty} E_{D C}=\frac{\lambda \pi^{2}}{\alpha^{2}} L P_{t} \csc \left(\frac{2 \pi}{\alpha}\right) \\
& \times\left(2 \alpha \gamma_{2}+12 \gamma_{4} \lambda \pi^{2} P_{t} \csc \left(\frac{2 \pi}{\alpha}\right)+9(\alpha-2) \frac{m+1}{m} P_{t} \gamma_{4}\right) .
\end{aligned}
$$




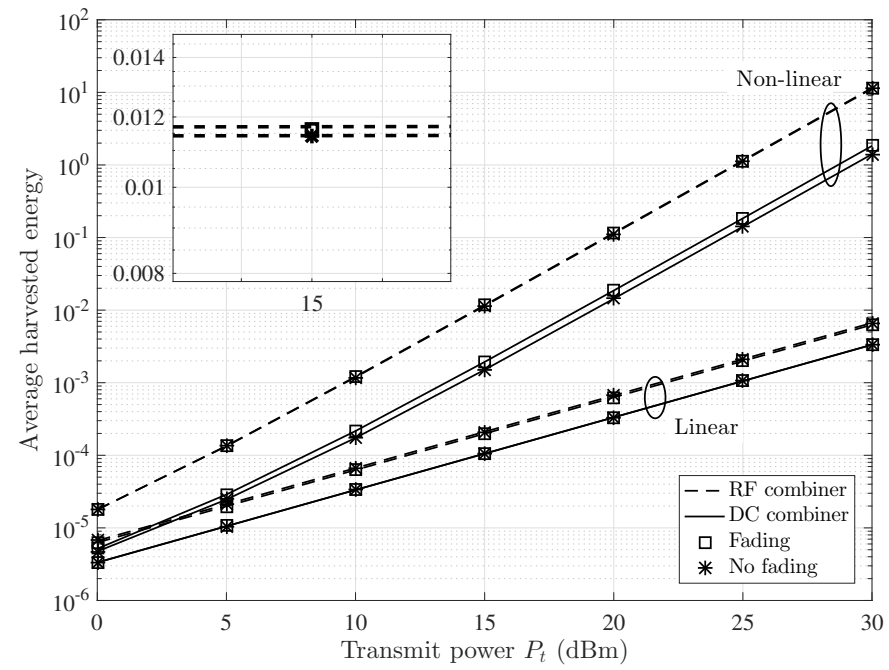

Fig. 1. Average harvested energy versus the transmit power; $m=2, L=2$.

Proof. See Appendix C.

The following remark considers the case $m \rightarrow \infty$, in other words, the case with no fading.

Remark 3. For large values of $m$, the average harvested energy asymptotically converges to

$$
\begin{aligned}
\lim _{m \rightarrow \infty} E_{R F}=P_{t} L^{2} \sum_{K=0}^{\infty} p(K) K\left(\gamma_{2} \bar{r}_{1}\right. & +3 P_{t} \gamma_{4} L^{2}(K-1) \bar{r}_{1}^{2} \\
& \left.+\frac{9}{2} P t \gamma_{4} L^{2} \bar{r}_{2}\right),
\end{aligned}
$$

for the RF combiner, and

$$
\begin{aligned}
\lim _{m \rightarrow \infty} E_{D C}=P_{t} L \sum_{K=0}^{\infty} p(K) K\left(\gamma_{2} \bar{r}_{1}\right. & +3 P_{t} \gamma_{4}(K-1) \bar{r}_{1}^{2} \\
& \left.+\frac{9}{2} P_{t} \gamma_{4} \bar{r}_{2}\right)
\end{aligned}
$$

for the DC combiner.

Proof. The expression for the RF combiner uses the fact that

$$
w_{i}=\frac{\Gamma(m+i / 2)}{\Gamma(m)} \rightarrow m^{i / 2},
$$

for very large $m$. Therefore, using the above, we have $e_{2}=L^{2}$ and $e_{4}=L^{4}$, and the result for the RF combiner follows. The expression for the DC combiner is derived simply from the fact that $(m+1) / m \rightarrow 1$ for large $m$.

It is clear from (22), that when the DC combiner is employed, fading is beneficial to the average harvested energy. Specifically, compared to (15), fading has no effect when considering the linear model (first term). However, when the non-linear model is considered, the third term in (15) is always greater than the one in (22) due to the $(m+1) / m$ factor. This is similar to the observations in [9] for $m=1$. On the other hand, when the RF combiner is employed, fading is not always beneficial but depends on the parameters $L$ and $m$.

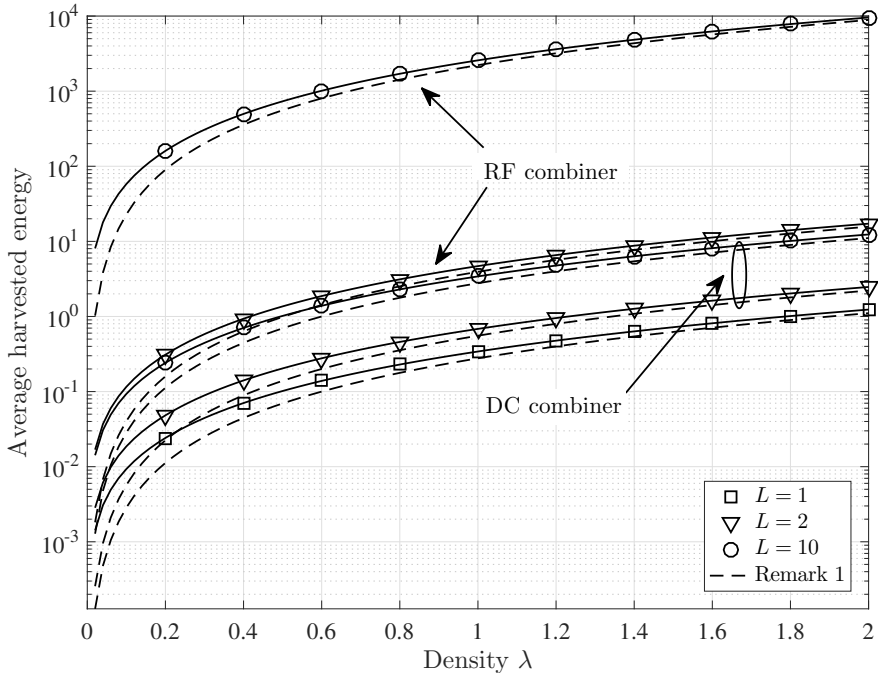

Fig. 2. Average harvested energy versus the density; $m=2, P_{t}=20 \mathrm{dBm}$.

\section{NumericAl Results}

We evaluate our proposed model through computer simulations. Unless otherwise stated, we use the following parameters: $\lambda=0.1, \rho=10 \mathrm{~m}, \alpha=4, \gamma_{2}=0.0034$ [9] and $\gamma_{4}=0.3829$ [9]. In the figures, theoretical results are depicted with lines whereas simulation results are depicted with markers.

Fig. 1 depicts the average harvested energy in terms of the transmit power $P_{t}$. The first main observation is that by considering the non-linearities of the energy harvesting process, the average harvested energy exhibits significant gains, compared to a linear approach (i.e. taking only the first term in (4)). Indeed, these gains become more important as the transmit power increases. Another clear observation, is that the RF combiner outperforms the $\mathrm{DC}$ combiner. This is due to the fact that a single diode is used for the RF combiner, so the single diode's output is higher that the sum over multiple diode outputs at the DC combiner (as a result of the non-linearities). However, it is important to note here that we assume both combiners have the same efficiency, whereas this might not be the case in practical scenarios. Fig. 1 also illustrates the performance of each scenario with and without fading (Remark 3). As noted in Section III, fading is beneficial for energy harvesting when non-linearities are taken into account and the DC combiner is employed. For the considered scenario, fading is also beneficial with the RF combiner but the gains are very small. Finally, the simulation results (markers) perfectly match the theoretical results (lines), which validate our analysis.

Fig. 2 illustrates the average harvested energy with respect to the network's density $\lambda$. As expected, the average harvested energy increases with the density, since a larger density implies a larger aggregate received signal at the device. The figure also shows the average harvested energy based on the expressions for ultra-dense networks in Remark 1. It is clear that the expressions are tight approximations to Propositions 1 and 


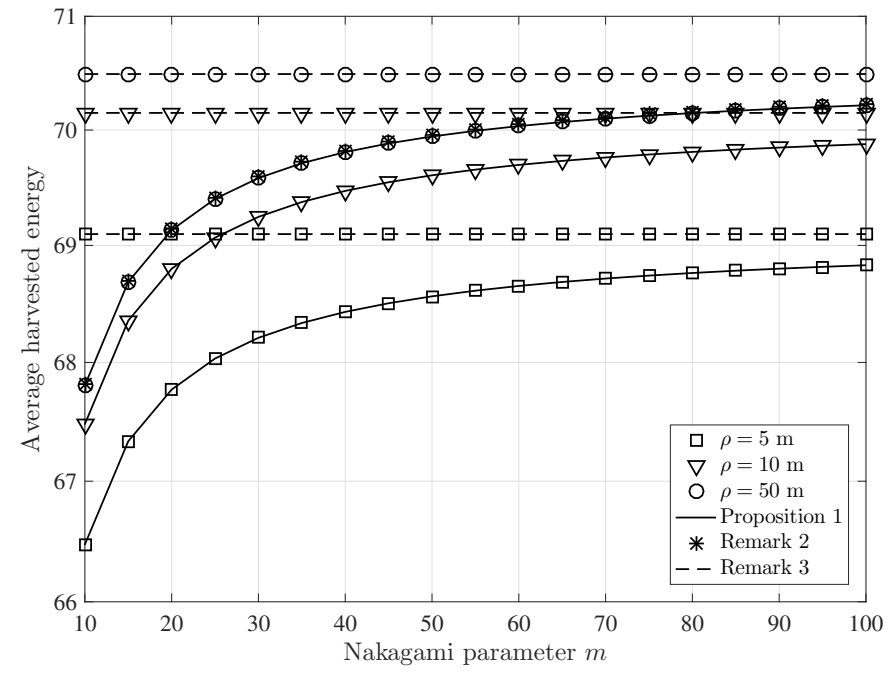

Fig. 3. Average harvested energy with the RF combiner versus the Nakagami parameter; $L=10, P_{t}=20 \mathrm{dBm}$.

2 for large $\lambda$, which validate our assumptions. Finally, the performance in terms of the rectenna array size is also shown. The average harvested energy increases with $L$, as expected. Furthermore, as shown in Fig. 1, the RF combiner outperforms the $\mathrm{DC}$ combiner for $L>1$. When $L=1$, we have $\mathrm{E}_{\mathrm{RF}}=\mathrm{E}_{\mathrm{DC}}$ since no combination takes place in this case.

Finally, Fig. 3 depicts the average harvested energy with the RF combiner versus the Nakagami fading parameter $m$. It's clear that the average harvested energy increases with $m$, which verifies our observations in Section III that fading is not always beneficial when the RF combiner is employed. As $m$ increases, the fading becomes deterministic and converges to the asymptotic case of Remark 3. The figure also shows how the cell's radius $\rho$ affects the harvested energy. As the radius increases, the harvested energy also increases due to the higher number of transmitters. This increment is more visible as the value of $m$ increases. However, the gains in harvested energy become smaller as $\rho$ increases. This is because the signals from the transmitters located at the edge of the cell undergo large propagation losses. Therefore, as $\rho$ increases, the average harvested energy will converge to a constant value. This is clear from the fact that for $\rho=50 \mathrm{~m}$, the average harvested energy has converged to the asymptotic case $\rho \rightarrow \infty$, given in Remark 2.

\section{CONCLusion}

In this paper, we focused on devices which harvest energy from ambient RF signals, underlaid in a wireless communication network. We considered the employment of rectenna arrays at the devices and have taken into account the nonlinearities of the energy harvesting process. With the use of tools from stochastic geometry, we derived closed-form expressions for the average harvested energy. Our results show the importance of considering non-linearities, mainly in terms of the network's density and the size of the rectifying antenna array but also for other network parameters.

\section{APPENDIX}

\section{A. Proof of Proposition 1}

In order to derive the average harvested energy when employing the RF combiner, we need to evaluate (7). We first focus on the second order term. Conditioned on the number of transmitters in $\mathcal{D}$ being $K$, we have

$$
\begin{aligned}
\mathbb{E}\left[y(t)^{2} \mid K\right] & =\mathbb{E}\left[\left(\sum_{k=1}^{K} y_{k}^{\mathrm{RF}}(t)\right)^{2}\right]=\sum_{k=1}^{K} \mathbb{E}\left[y_{k}^{\mathrm{RF}}(t)^{2}\right] \\
& =\sum_{k=1}^{K} P_{t} \mathbb{E}\left[\frac{1}{1+d_{k}^{\alpha}}\right] \mathbb{E}\left[c_{k}(t)^{2}\right] \\
& =K P_{t} \bar{r} \mathbb{E}\left[c_{k}(t)^{2}\right],
\end{aligned}
$$

where $y_{k}^{\mathrm{RF}}(t)$ is given by (6) and the expectation over the distance to the $k$-th transmitter is given by (10). The random variable $c_{k}(t)$ is the sum of $L$ independent and identically distributed Nakagami random variables with equal parameter $m$. From [12], we can accurately approximate its second moment by

$$
\begin{aligned}
\epsilon_{2}=\mathbb{E}\left[c_{k}(t)^{2}\right]=L(1 & +\frac{(L-1)}{m}\left(\frac{\Gamma(m+1 / 2)}{\Gamma(m)}\right)^{2} \\
& \left.\times{ }_{2} F_{1}\left(-\frac{1}{2},-\frac{1}{2} ; m ; \beta\right)\right),
\end{aligned}
$$

where $\beta$ is the correlation coefficient between each pair of the $L$ random variables. Then, since we have uncorrelated fading channels, the correlation coefficient is $\beta=0$ and so ${ }_{2} F_{1}\left(-\frac{1}{2},-\frac{1}{2} ; m ; 0\right)=1$, which gives (12). We now deal with the fourth order term, that is, we derive the following

$$
\mathbb{E}\left[y(t)^{4} \mid K\right]=\mathbb{E}\left[\left(\sum_{k=1}^{K} y_{k}^{\mathrm{RF}}(t)\right)^{4}\right] .
$$

Using the multinomial theorem, the fourth power of the sum can be expanded as

$$
\mathbb{E}\left[y(t)^{4} \mid K\right]=K \mathbb{E}\left[y_{k}^{\mathrm{RF}}(t)^{4}\right]+3 K(K-1)\left(\mathbb{E}\left[y_{k}^{\mathrm{RF}}(t)^{2}\right]\right)^{2},
$$

where $\mathbb{E}\left[y_{k}^{\mathrm{RF}}(t)^{4}\right]$ can be written as

$$
\begin{aligned}
\mathbb{E}\left[y_{k}^{\mathrm{RF}}(t)^{4}\right] & =\frac{3}{2} P_{t}^{2} \mathbb{E}\left[\frac{1}{\left(1+d_{k}^{\alpha}\right)^{2}}\right] \mathbb{E}\left[c_{k}(t)^{4}\right] \mathbb{E}\left[x(t)^{4}\right] \\
& =\frac{9}{2} P_{t}^{2} \bar{r}_{2} \mathbb{E}\left[c_{k}(t)^{4}\right]
\end{aligned}
$$

where (27) follows from $\mathbb{E}\left[\cos ^{4} \theta_{k}\right]=3 / 8$ for a uniformly distributed phase $\theta_{k}$ and (28) follows from $\mathbb{E}\left[|x(t)|^{4}\right]=3$ and from the derived expression in (10). The fourth moment of $c_{k}(t)$ can be evaluated from [12] and setting the correlation coefficient to zero, which gives (13). Finally, by replacing (24), (26) and (28) in (4) and by using the PMF given by (9) to uncondition on the number of transmitters $K$, the results follows. 


\section{B. Proof of Proposition 2}

The proof follows similar steps is the proof of Proposition 1. The main difference here is that the signals are combined at the DC domain. Specifically, for the DC combiner case, (24) is written as

$$
\mathbb{E}\left[y(t)^{2} \mid K\right]=K P_{t} \bar{r} \mathbb{E}\left[\sum_{i=1}^{L}\left|h_{k, i}(t)\right|^{2}\right],
$$

where $\left|h_{k, i}(t)\right|^{2}$ is a Gamma random variable with shape and scale parameters $m$ and $1 / m$, respectively. As $\left|h_{k, i}(t)\right|^{2}$ are independent and identically distributed with equal scale parameter,

$$
\mathbb{E}\left[\sum_{i=1}^{L}\left|h_{k, i}(t)\right|^{2}\right]=\sum_{i=1}^{L} \mathbb{E}\left[\left|h_{k, i}(t)\right|^{2}\right]=L,
$$

which follows from the first moment of a Gamma random variable. Similarly, (28) for the DC combiner case is given by

$$
\begin{aligned}
\mathbb{E}\left[y_{k}^{\mathrm{RF}}(t)^{4}\right] & =\frac{9}{2} P_{t}^{2} \bar{r}_{2} \mathbb{E}\left[\sum_{i=1}^{L}\left|h_{k, i}(t)\right|^{4}\right] \\
& =\frac{9}{2} P_{t}^{2} \bar{r}_{2} \frac{L(m+1)}{m},
\end{aligned}
$$

which follows from the second moment of a Gamma random variable.

\section{Proof of Remark 2}

In the case of a large cell, i.e. $\rho \rightarrow \infty$, the received signal $y(t)$ is an infinite sum over the point process $\Phi$. We will consider the proof for the RF combiner and omit the proof for the DC combiner, as it follows similar steps. Therefore, we need to derive the second and fourth moment of

$$
y(t)=\sum_{x_{k} \in \Phi} y_{k}^{\mathrm{RF}}(t) .
$$

The second moment is given by

$$
\begin{aligned}
& \mathbb{E}\left[y(t)^{2}\right]=\mathbb{E}\left[\left(\sum_{x_{k} \in \Phi} y_{k}^{\mathrm{RF}}(t)\right)^{2}\right]=\mathbb{E}\left[\sum_{x_{k} \in \Phi} P_{t} \frac{c_{k}(t)^{2}}{1+d_{k}^{\alpha}}\right] \\
& =P_{t} \epsilon_{2} 2 \pi \lambda \int_{0}^{\infty} \frac{r}{1+r^{\alpha}} d r=\frac{2}{\alpha} P_{t} \epsilon_{2} \lambda \pi^{2} \csc \left(\frac{2 \pi}{\alpha}\right),
\end{aligned}
$$

where $\csc (\cdot)$ is the cosecant function, $\mathbb{E}\left[c_{k}(t)^{2}\right]$ is derived as in (25) and the expectation over the path-loss is derived using Campbell's theorem [13] and by applying [11, 3.241.2] with the transformation $r^{2} \rightarrow u$. For the fourth moment, we have

$$
\begin{aligned}
\mathbb{E}\left[\left(\sum_{x_{k} \in \Phi} y_{k}^{\mathrm{RF}}(t)\right)^{4}\right] & =\mathbb{E}\left[\sum_{x_{k} \in \Phi} y_{k}^{\mathrm{RF}}(t)^{4}\right] \\
+3 \mathbb{E} & {\left[\sum_{\substack{x_{k_{1}}, x_{k_{2}} \in \Phi \\
k_{1} \neq k_{2}}} y_{k_{1}}^{\mathrm{RF}}(t)^{2} y_{k_{2}}^{\mathrm{RF}}(t)^{2}\right], }
\end{aligned}
$$

which follows from the multinomial theorem. The first term can be written as

$$
\begin{aligned}
\mathbb{E}\left[\sum_{x_{k} \in \Phi} y_{k}^{\mathrm{RF}}(t)^{4}\right] & =\frac{9}{2} P_{t}^{2} \epsilon_{4} 2 \pi \lambda \int_{0}^{\infty} \frac{r}{\left(1+r^{\alpha}\right)^{2}} d r \\
& =9 P_{t}^{2} \epsilon_{4} \lambda \pi^{2} \frac{(\alpha-2)}{\alpha^{2}} \csc \left(\frac{2 \pi}{a}\right),
\end{aligned}
$$

where the expectations follow the same arguments as before and the integral is evaluated with the help of $[11,3.241 .5]$. The expectation in the second term can be written as [13]

$$
\begin{aligned}
\mathbb{E}\left[\sum_{\substack{x_{k_{1}}, x_{k_{2}} \in \Phi \\
k_{1} \neq k_{2}}} y_{k_{1}}^{\mathrm{RF}}(t)^{2} y_{k_{2}}^{\mathrm{RF}}(t)^{2}\right] & =\mathbb{E}\left[\sum_{x_{k_{1}} \in \Phi} y_{k_{1}}^{\mathrm{RF}}(t)^{2}\right] \\
& \times \mathbb{E}\left[\sum_{x_{k_{2}} \in \Phi} y_{k_{2}}^{\mathrm{RF}}(t)^{2}\right],
\end{aligned}
$$

which is the second moment, given by (33), squared. Finally, the final expressions is deduced by substituting the derived second and fourth moments in (4).

\section{REFERENCES}

[1] Y. Zeng, B. Clerckx, and R. Zhang, "Communications and signals design for wireless power transmission," IEEE Trans. Commun., vol. 65, no. 5, pp. 2264-2290, May 2017.

[2] I. Flint, X. Lu, N. Privault, D. Niyato, and P. Wang, "Performance analysis of ambient RF energy harvesting with repulsive point process modeling," IEEE Trans. Wireless Commun., vol. 14, pp. 5402-5416, Oct. 2015.

[3] A. H. Sakr and E. Hossain, "Analysis of $K$-tier uplink cellular networks with ambient RF energy harvesting," IEEE J. Sel. Areas Commun., vol. 33, pp. 2226-2238, Oct. 2015.

[4] M. A. Kishk and H. S. Dhillon, "Joint uplink and downlink coverage analysis of cellular-based RF-powered IoT network," IEEE Trans. Green Commun. Netw., vol. 2, pp. 446-459, Dec, 2017.

[5] T. X. Doan, T. M. Hoang, T. Q. Duong, and H. Q. Ngo, "Energy harvesting-based D2D communications in the presence of interference and ambient RF sources," IEEE Access, vol. 5, pp. 5224-5234, Mar. 2017.

[6] S. Kusaladharma and C. Tellambura, "Performance characterization of spatially random energy harvesting underlay D2D networks with transmit power control," IEEE Trans. Green Commun. Netw., vol. 2, pp. 87-99, Mar. 2018.

[7] D. T. Hoang, D. Niyato, P. Wang, D. I. Kim, and Z. Han "Ambient backscatter: A new approach to improve network performance for RFpowered cognitive radio networks," IEEE Trans. Commun., vol. 65, pp. 3659-3674, Sept. 2017.

[8] X. Lu, H. Jiang, D. Niyato, D. I. Kim, and Z. Han, "Wirelesspowered device-to-device communications with ambient backscattering: Performance modeling and analysis," IEEE Trans. Commun., vol. 17, pp. 1528-1544, Mar. 2018.

[9] B. Clerckx and J. Kim, "On the beneficial roles of fading and transmit diversity in wireless power transfer with nonlinear energy harvesting," IEEE Trans. Wireless Commun., vol. 17, pp. 7731-7743, Sept. 2018.

[10] U. Olgun, C.-C. Chen, and J. L. Volakis, "Investigation of rectenna array configurations for enhanced RF power harvesting," IEEE Ant. Wireless Prop. Lett., vol. 10, pp. 262-265, Apr. 2011.

[11] I. S. Gradshteyn and I. M. Ryzhik, Table of Integrals, Series, and Products. Elsevier, 2007.

[12] Z. Hadzi-Velkov, N. Zlatanov, and G. K. Karagiannidis, "An efficient approximation to the correlated Nakagami- $m$ sums and its application in equal gain diversity receivers," IEEE Trans. Wireless Commun., vol. 9, pp. 302-310, Jan. 2010.

[13] J. F. C. Kingman, Poisson Processes, Oxford University Press, 1993. 